\title{
Revisión de la Problemática de la Calidad del Software para el Desarrollo de Aplicaciones de Computación en la Nube
}

\author{
Carlos A. Guerrero(1)* $y$ Jorge M. Londoño(2) \\ (1) Unidades Tecnológicas de Santander, Calle de los Estudiantes \# 9-82 Ciudadela Real de Minas, \\ Bucaramanga, Santander - Colombia. (e-mail: anguerrco@msn.com) \\ (2) Universidad Pontificia Bolivariana - UPB, Campus de Laureles Circular 1 No. 70-01, Medellín, Antioquia \\ -Colombia. (e-mail: jorge.londono@upb.edu.co)
}

${ }^{*}$ Autor a quien debe ser dirigida la correspondencia

Recibido Sep. 15, 2015; Aceptado Nov. 10, 2015; Versión final Feb. 10, 2016, Publicado Jun. 2016

\begin{abstract}
Resumen
El objetivo de este trabajo es apoyar los procesos de investigación y el desarrollo de aplicaciones en la nube con calidad de software aceptable. Se presenta la caracterización de los trabajos realizados entre los años de 2010 y 2014, utilizando como metodología la selección de fuentes primarias de alto impacto y la revisión exhaustiva y crítica de las fuentes bibliográficas encontradas. Se seleccionaron 206 trabajos, los cuales fueron clasificados en 8 áreas. El área de interés de este estudio es la calidad, y por tal motivo se realizó una clasificación que permitió encontrar 5 subcategorías: calidad del servicio, calidad en el proceso de desarrollo, calidad computación móvil en la nube, calidad de los datos y calidad de la experiencia. Se concluye que la calidad de los datos y la privacidad de los mismos siguen y seguirán siendo un tema crítico en los niveles de calidad de servicio.
\end{abstract}

Palabras clave: computación en la nube; calidad de software; servicios de software; arquitectura.

\section{A survey of software quality for the development of cloud computing applications}

\begin{abstract}
The objective of this study is to provide an in depth review of the current research about the process and the development of quality cloud applications. Papers published between 2010 and 2014 were thoroughly analyzed following a methodology of collecting primary sources of high impact and the exhaustive and critical analysis of the selected bibliographic sources. Of all sources found, 206 papers were selected and classified in eight areas. Being the quality of the software development process, the main area of interest in this work, a classification that allowed identifying five key subcategories was done: quality of service, development process quality, mobile cloud compute quality, data quality, and quality of experience. In is concluded that quality data and privacy of such data are and they will continue being critical subjects on the quality of software services.
\end{abstract}




\section{INTRODUCCIÓN}

La computación en la nube es un paradigma que almacena la información en servidores de Internet y se utiliza desde clientes con almacenamiento temporal, incluye el uso de equipos de escritorio, tabletas, portátiles, etc. Es un modelo que permite al usuario acceder a servicios estandarizados para responder a sus necesidades de manera adaptativa, rápida y flexible, pagando únicamente por el consumo realizado. Este concepto puede ser abordado desde tres puntos de vista: en primer lugar se observa que la computación en la nube es el resultado de la evolución de un conjunto de tecnologías que se han consolidado desde hace varios años; en segundo lugar, es una tendencia tecnológica que goza de gran popularidad y está siendo ampliamente adoptada; en tercer lugar, y el más importante en el marco de este trabajo, en el área de desarrollo orientado a la computación en la nube se siguen utilizando las mismas metodologías de los procesos de desarrollo tradicionales.

Ahora bien, la consolidación de la computación en la nube en el mundo, ha traído como consecuencia la aparición de conceptos y definiciones que permiten clasificar cada servicio que se puede ofrecer a través de este medio; la forma de identificar estos servicios es utilizando el sufijo as a service - aas, es así como se pueden encontrar términos como SaaS (Software as a Service) (Hou y Zhou, 2010), PaaS (Platform as a Service) (Ardagna et al., 2012), TaaS (Tools as a Service) (Yu et al., 2010), laaS (Infrastructure as a Service) (Guo, 2003), KaaS (knowledge as a Service) (Abdullah et al., 2011) por mencionar algunos. La computación en la nube es más una evolución tecnológica, que un concepto novedoso. Entre las principales evidencias históricas se encuentran; primero, el uso que empresas y universidades hacían de las grandes computadoras centrales, lo que hoy en día se conoce como servicio de accesibilidad remota a los recursos de procesamiento de otra computadora y segundo, la aparición de las redes privadas virtuales VPN (Virtual Private Network) (Liao y Su, 2011), los servicios ofrecidos a través de esta topología de red se extrapolan a los servicios que hoy en día se ofrecen a través de la computación en la nube. Gracias a los servicios de la computación en la nube la tendencia actual está orientada a contratar servicios externos en centros de datos ubicados en cualquier parte del mundo.

Las ideas presentadas anteriormente resaltan la importancia de realizar estudios sobre la computación en la nube que permitan definir una hoja de ruta para realizar trabajos de investigación en esta temática, Singh y Chana (2012) elaboraron un estado del arte en el artículo Cloud Based Development Issues: A Methodical Analysis, el cual estableció un estado del arte inicial para los temas más relevantes de y computación en la nube, entre los que resalta: 1) arquitectura, 2) desarrollo basado en componentes y reutilización, 3) calidad del servicio, 4) diseño y 5) seguridad. El presente estudio profundiza en el análisis de la calidad del software, desde el punto de vista del proceso de ingeniería para el desarrollo de aplicaciones orientadas a entornos de nube y se proponen nuevas categorías para tener en cuenta aspectos que no fueron considerados en el trabajo de Singh y Chana (2012).

La amplia revisión de fuentes permitió establecer una categorización más exhaustiva de los criterios para abordar la temática de computación en la nube, el objeto del presente artículo es describir los aspectos relevantes del área "Calidad", con el fin de proporcionar herramientas de decisión para los ingenieros de desarrollo que se inclinen por trabajar con esta tendencia tecnológica. La razón principal para seleccionar el área de calidad, está dada por la importancia que tienen las características de esta área en un proceso de desarrollo maduro y robusto. Por tal motivo, la orientación de esta revisión se enfocó en la definición y descripción de los indicadores de cada característica que afecta la calidad de los desarrollos de la computación en la nube.

El artículo presentado a continuación inicia con la descripción de la metodología utilizada en el proceso de selección de trabajos sobre calidad en el desarrollo de software para computación en la nube, además, presenta los criterios y fuentes primarias utilizadas en el proceso de búsqueda. A continuación, se encuentran los hallazgos y una clasificación de los trabajos empleada para realizar un análisis por categorías de todas las fuentes recopiladas. Posteriormente se presenta el análisis de las cinco subcategorías identificadas en el área de calidad en el desarrollo de aplicaciones para computación en la nube. Finalmente, se describen las posibles amenazas del estudio, así como las conclusiones y referencias.

\section{METODOLOGÍA DE INVESTIGACIÓN UTILIZADA}

El análisis de los estudios se realizó en dos etapas, en la primera se seleccionaron referencias bibliográficas de las fuentes más representativas: 1) IEEE Explore, 2) ACM Digital Library y 3) Springer LNCS. Esta revisión se realizó utilizando palabras claves orientadas a temas relacionados con computación en la nube, como se detallan más adelante. Los resultados del estudio se clasificaron en cinco subcategorías que le permiten al lector identificar los avances en cada de una de ellas. 
En la segunda etapa se realizó un proceso de búsqueda más extenso, ampliando el rango a revistas categorizadas por la métrica de impacto de Scopus SJR - SCimago Journal Rank, (ScimagoLab,2014) e incluyendo en la búsqueda palabras claves que permitieron tener una clasificación completa de los estudios realizados para computación en la nube. La ventana de observación definida para este estudio fue de cuatro años, iniciando en el año 2010. A continuación, se presenta el detalle de las actividades realizadas en cada una de las etapas:

\section{Búsqueda}

Etapa I: Como se enunció anteriormente las tres fuentes primarias fueron: IEEE Explore, ACM Digital Library y Springer LNCS. Teniendo en cuenta la gran cantidad de información existente en la temática relacionada con la computación en la nube y los estudios previos realizados por otros autores, ésta etapa acotó el estudio solo a trabajos en donde la temática se relacionará con la calidad en la computación en la nube. Los términos utilizados en el proceso de búsqueda se relacionan en la Tabla 1, así como sus variaciones.

Tabla 1: Palabras claves de búsqueda en la etapa I

\begin{tabular}{|l|l|}
\hline \multicolumn{1}{|c|}{ Palabras clave } & \multicolumn{1}{c|}{ Variaciones } \\
\hline Quality Cloud & Quality Cloud Software engineering \\
\hline Global software development & Quality global software development \\
\hline Quality Cloud Model & Quality Model \\
\hline Cloud Web services & Cloud software \\
\hline Patterns cloud computing & Design patterns cloud \\
\hline
\end{tabular}

Después de realizar la búsqueda y recopilar los artículos, se realizó un análisis preliminar de las palabras claves encontradas en los estudios para establecer relaciones entre los mismos, se encontraron en esta primera etapa 146 palabras claves diferentes a los criterios de búsqueda utilizados, entre las más representativas están: quality of service, test-bed, software quality management, cloud computing, cloud database, cloud service, interoperability, architecture, data mining, data quality engineering, service level agreement, mentoring, entre otras.

Etapa II: En esta etapa se realizó una búsqueda de revistas con índice de impacto de acuerdo a la métrica del SCImago Journal \& Country Rank en países con publicaciones en el área de las ciencias computacionales. Utilizando ciencias computacionales como criterio de búsqueda, se encontraron una gran cantidad de revistas las cuales fueron verificadas una a una en todos los volúmenes que coincidieron con la ventana de observación. La verificación tuvo por objeto la identificación de artículos con temáticas de computación en la nube. La estrategia de las palabras claves de la etapa I se repitió incluyendo una palabra clave más genérica computación en la nube en tres idiomas: inglés, español y portugués.

\section{Hallazgos}

El proceso de tabulación de la información de los artículos permitió obtener los resultados que se relacionan en este apartado. La Fig. 1 presenta los países de origen de las revistas en donde se encontraron los artículos, se presentan los países con total de artículos igual o superior a 5 pues corresponden al $10 \%$ del mayor valor encontrado.

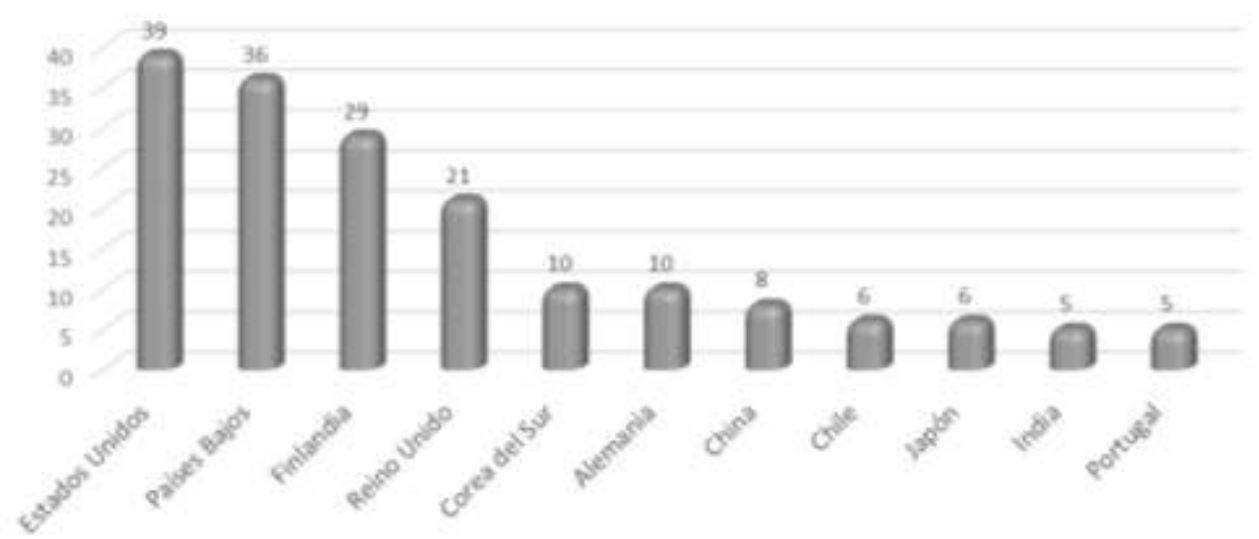

Fig. 1: Cantidad de artículos publicados en revistas por país 
El país con mayor número de publicaciones en sus revistas en temas de computación en la nube es Estados Unidos. Los países con un número de artículos inferior a 5 que no se visualizan en la Fig. 1, fueron: Italia, Australia, Croacia, Grecia, Egipto, Malasia, Rumania, Irlanda, Taiwán, Shanghái, Inglaterra, Turquía, Sur África, Brasil, España, Jordania, México, Hawái, Francia y Lituania.

A pesar de ser Estados Unidos el país con más publicaciones en temas de computación en la nube, las instituciones a la que están afiliados los autores que más publican estudios en este tema, son de origen chino. En la Fig. 2 se pueden apreciar los países de las instituciones origen a la que están afiliados los autores que publican alrededor de esta temática en revistas y eventos a nivel mundial.

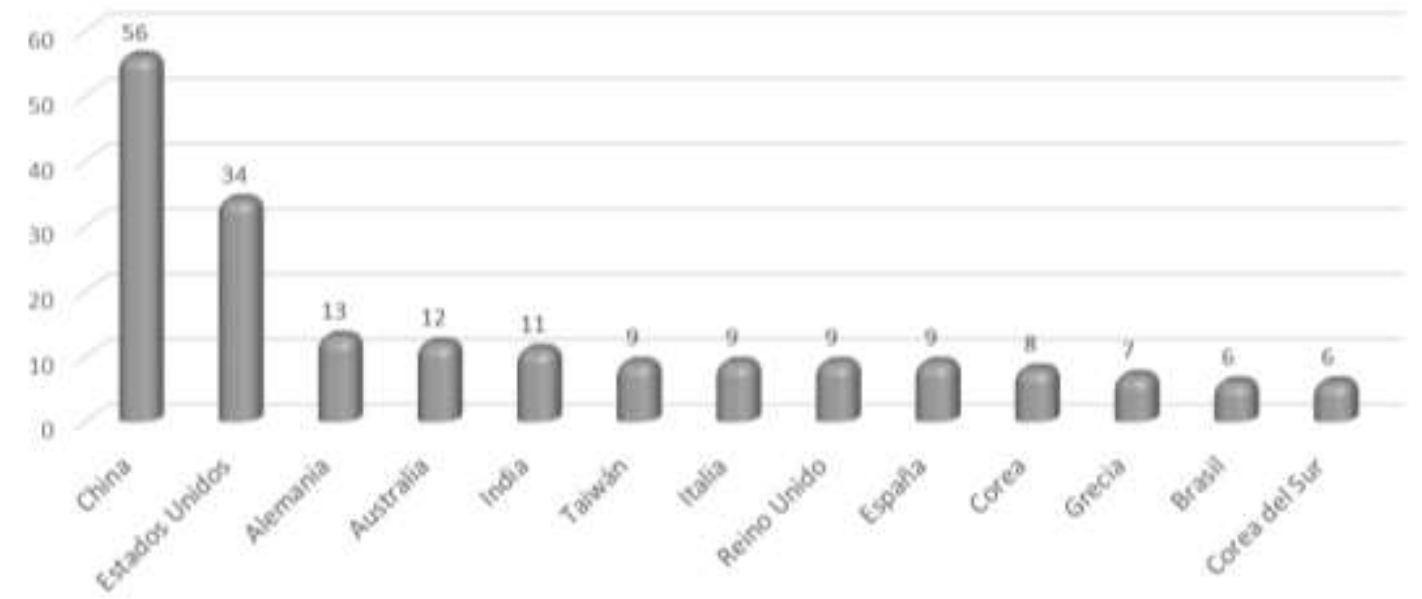

Fig. 2: Cantidad de artículos por país de origen del estudio

En la ventana de observación analizada, se presentó un aumento significativo durante los cuatro primeros años en publicaciones con temáticas relacionadas a la computación en la nube. La Fig. 3, presenta las cantidades de artículos publicados por año. El corte del año 2014 se realizó en el mes de julio, razón por la cual solo encontraron 5 trabajos.

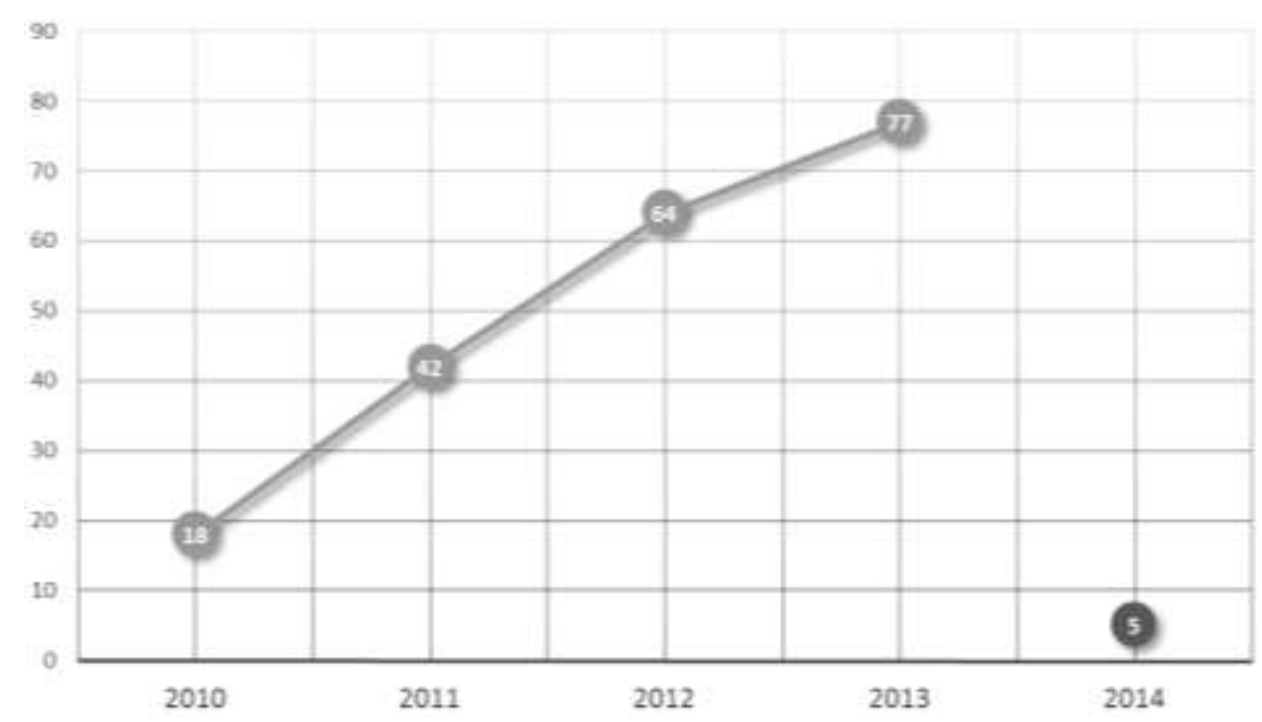

Fig. 3: Evolución estudios Computación en la Nube

El presente estudio identificó 206 artículos de alto impacto con temas relacionados con la computación en la nube, de la lectura de los mismos se pudo establecer una primera clasificación de los artículos, los cuales quedaron agrupados en 8 áreas: 1) infraestructura como servicio, 2) calidad, 3) seguridad, 4) conceptualización, 5) software como servicio, 6) caso aplicado, 7) acuerdos de niveles de servicio y 8) móviles.

Un estudio previo acerca de la problemática del desarrollo basado en la nube, realizado por Singh y Chana (2012), propuso la siguiente clasificación: 1) arquitectura, 2) desarrollo basado en componentes (DBS) y reusabilidad, 3) calidad del servicio, 4) diseño y 5) seguridad. El presente estudio propone nuevas categorías para tener en cuenta aspectos que no fueron considerados en el trabajo de Singh y Chana (2012). En la Tabla 2 se presenta una correlación entre la clasificación del estudio base y el estudio actual, así como las nuevas categorías que resultan de este estudio. 
Como se puede observar en la Tabla 2, la clasificación Infraestructura como servicio denominada Architecture en el estudio previo, continúa como primera en la lista. La clasificación propuesta para Calidad cubre dos tópicos del estudio previo: $C B D$ and Reusability y QoS (Quality of Service), que coindicen en ser temas de amplia relevancia ocupando el segundo puesto con mayor número de trabajos encontrados. Por su parte, la clasificación Seguridad ascendió al tercer puesto en el presente estudio. El gran número de trabajos encontrados y analizados en la ventana de observación, permitió establecer una clasificación acorde a las temáticas encontradas en cada uno de los estudios. La Fig. 4 presenta la clasificación inicial realizada en el presente estudio, así como el número de trabajos encontrados.

El objeto de estudio del presente trabajo son las publicaciones sobre calidad en la computación en la nube, por lo tanto, la distribución por áreas presentada en la Fig. 4. permitió acotar la muestra para realizar un análisis posterior más exhaustivo de cada uno de los trabajos. Así, del total de 206 estudios, se pasó a un grupo menor de 50 que aborda específicamente la temática de calidad en la computación en la nube.

Tabla 2: Clasificación de las problemáticas encontradas, alcance y relación con un estudio previo

\begin{tabular}{|c|c|c|}
\hline $\begin{array}{l}\text { Clasificación del } \\
\text { presente estudio }\end{array}$ & $\begin{array}{l}\text { Clasificación por } \\
\text { Singh y Chana (2012) }\end{array}$ & Alcance de la clasificación para los dos estudios \\
\hline $\begin{array}{l}\text { 1) Infraestructura y } \\
\text { plataformas como } \\
\text { servicio }\end{array}$ & 1) Arquitectura & $\begin{array}{l}\text { Hace referencia a la estructura y los recursos de los sistemas en la } \\
\text { nube, comprende servicios, software de comunicación entre } \\
\text { aplicaciones (middleware) y aspectos de software. }\end{array}$ \\
\hline 2) Calidad & $\begin{array}{l}\text { 2) Desarrollo basado } \\
\text { en componentes y } \\
\text { reusabilidad } \\
\text { 3) Calidad del servicio }\end{array}$ & $\begin{array}{l}\text { Se incluyen los temas relacionados con rendimiento, pruebas } \\
\text { funcionales, calidad del servicio ofrecido, calidad en los } \\
\text { requerimientos del negocio, calidad en la ejecución de programas, } \\
\text { paralelismo, seguimiento a los servicios, modelos de medición de } \\
\text { servicios, procesos de desarrollo, reutilización y calidad en los } \\
\text { datos. }\end{array}$ \\
\hline 3) Seguridad & 5) Seguridad & $\begin{array}{l}\text { En primera instancia se incluyen los temas de seguridad y } \\
\text { privacidad, cumplimiento y los acuerdos legales. En segunda } \\
\text { instancia se encuentran los temas relacionados con problemas de } \\
\text { seguridad en proveedores y los que enfrentan los clientes y en } \\
\text { tercera instancia, los problemas que se presentan a nivel de } \\
\text { seguridad al utilizar la infraestructura como servicio. }\end{array}$ \\
\hline 4) Conceptualización & --- & $\begin{array}{l}\text { Esta clasificación incluye los temas que permiten definir aspectos } \\
\text { conceptuales en la calidad de la computación en la nube, por } \\
\text { ejemplo: definiciones, ventajas y desventajas, servicios en la nube, } \\
\text { arquitectura base, entre otros. }\end{array}$ \\
\hline $\begin{array}{l}\text { 5) Software como } \\
\text { servicio }\end{array}$ & 4) Diseño & $\begin{array}{l}\text { Temas relacionados con la selección de servicios por parte de } \\
\text { clientes, sistemas de búsqueda con tolerancia a fallos, } \\
\text { requerimientos y retos del software como servicio y algoritmos para } \\
\text { el aprovisionamiento de recursos en la prestación de servicios. }\end{array}$ \\
\hline 6) Caso aplicado & --- & $\begin{array}{l}\text { Incluye los temas que presentan resultados de aplicaciones reales } \\
\text { que generan datos relevantes para soportar análisis y mediciones. }\end{array}$ \\
\hline $\begin{array}{l}\text { 7) Acuerdos de } \\
\text { niveles de servicio }\end{array}$ & --- & $\begin{array}{l}\text { Acuerdos de servicio entre clientes y proveedores, modelos y } \\
\text { métricas para cuantificar violaciones en los acuerdos. }\end{array}$ \\
\hline 8) Móviles & --- & $\begin{array}{l}\text { Hace referencia al uso de la nube para aplicaciones móviles, } \\
\text { inconvenientes en el desarrollo, análisis de los recursos limitados } \\
\text { de un móvil y la ventaja de usar la nube. }\end{array}$ \\
\hline
\end{tabular}

\section{Análisis de las subcategorías definidas para calidad en computación en la nube}

El grupo conformado por los 50 estudios se clasificó en 5 subcategorías del área calidad en la computación en la nube: 1) calidad del servicio, 2) calidad en el proceso de desarrollo, 3) calidad computación móvil en la nube, 4) calidad de los datos y 5) calidad de la experiencia. Estos estudios se distribuyen como se presenta en la Fig. 5. 


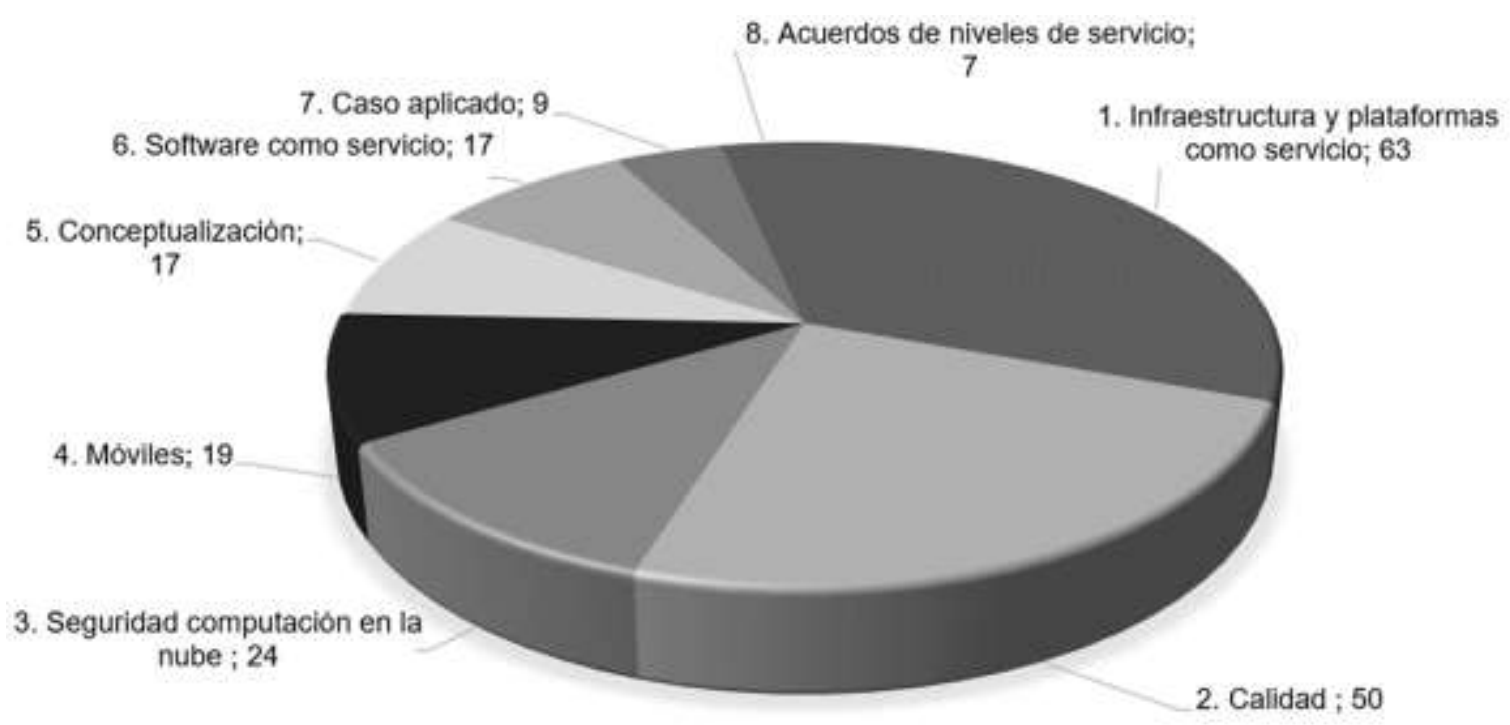

Fig. 4: Distribución de áreas Computación en la Nube

4. Calidad de los datos; 4

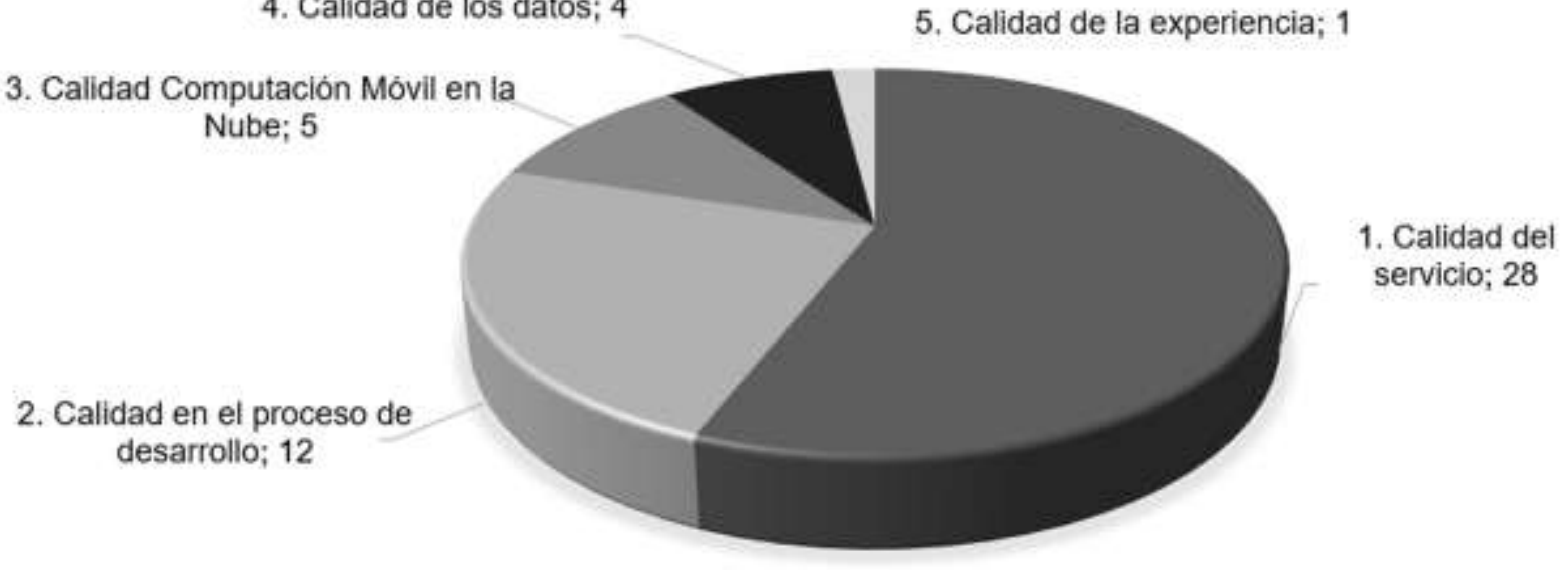

Fig. 5: Distribución de subcategorías de Calidad en la Computación en la Nube

La clasificación presentada anteriormente permitió identificar cuáles son las subcategorías con mayor cantidad de trabajos en el tema de calidad en computación en la nube. El tema de calidad del servicio es el de mayor interés debido a la necesidad latente por encontrar alternativas y estrategias que permitan brindar más y mejores servicios, buscando la satisfacción de las necesidades de los clientes y usuarios potenciales. En segundo lugar, se encuentra la subcategoría de calidad en el proceso de desarrollo, la cual aborda temas relacionados con la estrategia para el desarrollo global de aplicaciones. La subcategoría calidad computación móvil ocupa el tercer puesto en la clasificación, en donde priman los modelos de calidad para el software en dispositivos móviles. En el cuarto y quinto lugar se encuentran la calidad de los datos y la calidad de la experiencia. Para el contexto de este estudio, las cinco subcategorías identificadas en el área de calidad en la computación en la nube, permiten establecer los problemas relevantes, alrededor de los cuales se centran los esfuerzos de la comunidad científica en esta área.

En la siguiente sección se presenta cada subcategoría, se relacionan los trabajos encontrados en cada una de ellas y se presenta la temática principal de los mismos.

\section{RESULTADOS Y DISCUSIÓN}

\section{Calidad de la experiencia}

Hobfeld et al. (2012) abordan la problemática que se presenta en la gestión de la calidad de la experiencia en las aplicaciones en la nube. Las principales debilidades identificadas son la falta de lineamientos o modelos que permitan medir la percepción de los usuarios finales en contraste con las características que permiten medir la calidad del servicio -QoS-. De acuerdo con los autores, los principales factores que están involucrados en el ecosistema de la nube son: laaS, PaaS, SaaS, proveedores de servicios de internet, las redes de 
telecomunicaciones y finalmente la percepción del usuario final. No obstante, éstos se encuentran desarticulados y dificultan la gestión de la QoE (Quality of Experience). Para gestionar la calidad de la experiencia proponen tres pasos básicos: a) entender y modelar QoE: hace referencia a la definición de los parámetros que se deben tener en cuenta y deben asociarse directamente con las opiniones de los usuarios finales, b) monitorear y estimar QoE: se deben tener en cuenta la red, los equipos terminales, SLA (Service Level Agreement) con los operadores de servicios y la información específica sobre el servicio y las aplicaciones. Se requiere además el mapeo de características entre QoS y QoE. Finalmente, c) adaptar y controlar QoE para que el usuario no abandone el servicio por insatisfacción, tomando como referencia el análisis y control de las reacciones del usuario final. Los autores concluyen que, para garantizar altos índices en la QoE, se requiere de soluciones integrales que mapeen las características de QoE con las características de QoS. Los autores resaltan la importancia de interfaces abiertas y estandarizadas, debido a que teniendo presente esas características en los desarrollos en la nube se puede garantizar contenidos acordes a los contextos, así como eficiencia y minimización de costos con la adopción apropiada de este tipo de aplicaciones.

\section{Calidad de los datos}

Esta temática ha sido abordada por diversos estudios (Klems et al., 2012, Yang y Jia, 2011 y Zrnec y Lavbič, 2011) desde dos perspectivas: por un lado aspectos no funcionales como el rendimiento y la disponibilidad de los datos; y por el otro, la selección de estrategias para decidir la forma más apropiada de almacenar la información.

Klems et al. (2012) presentan un modelo para la medición de capacidades de almacenamiento de bases de datos en la nube. Las variables utilizadas fueron: rendimiento y disponibilidad. Yang y Jia (2011) orientan su estudio hacia la revisión de protocolos de auditoría para el seguimiento al almacenamiento de información en la nube. Igualmente plantean los requisitos necesarios para diseñar un protocolo de auditoría que permita realizar seguimiento a la información almacenada. El estudio finaliza describiendo retos desafiantes que pueden surgir al momento de diseñar un protocolo de auditoria eficiente para los datos almacenados en la nube. Los retos hacen referencia a tres formas diferentes de realizar una auditoría, auditoría dinámica, auditoría de colaboración y auditoría por lotes.

Zrnec y Lavbič (2011) se enfocan en revisar cuál es la estrategia más adecuada para el almacenamiento de la información: la forma tradicional o en la nube. Para esto establecen una matriz de comparación con base en dos criterios: rendimiento y costos. Los autores no concluyen cual es la mejor técnica de almacenamiento y proponen realizar una revisión más exhaustiva del tema pensando en las necesidades de cada organización. En consecuencia, si no se tiene en cuenta el contexto no hay garantía de que el método funcione. La conclusión principal de este trabajo, evidencia la necesidad de analizar más variables que permitan decidir si el almacenamiento en la nube es la opción definitiva para las copias de seguridad empresariales.

Finalmente, en esta subcategoría se encuentra el trabajo realizado por Comerio et al. (2010), según estos autores, las técnicas tradicionales de ingeniería de calidad de datos, son usadas y empleadas sin tener en cuenta el ambiente empresarial sobre el cual se va aplicar, esto tiene como consecuencia el deterioro en la calidad de los datos. Con el fin de mejorar las técnicas de ingeniería de calidad de datos, proponen el uso de tres modelos como: Software como Servicio, Datos como un servicio (DaaS) y la colaboración abierta distribuida (crowdsourcing), estos tres modelos son los que se encuentran disponibles en la nube para analizar la ingeniería de calidad de los datos (DQE por Data Quality Engineer) y calidad de la publicación de datos (QDP por Data Quality Publication) y permiten clasificaciones y asociaciones de las actividades de las fases de DQE y QDP. Este trabajo también analiza los requerimientos de los procesos de DQE y QDP en la nube, para luego proponer una arquitectura conceptual que de soporte a las técnicas de ingeniería de datos y publicación por medio de los modelos indicados anteriormente.

\section{Calidad Computación Móvil en la Nube}

Los trabajos realizados por Franke et al. (2012), Alnanih et al. (2013) y La y Kim (2013) abordan ésta temática definiendo modelos de calidad para la computación móvil en la nube. Para esto definen atributos tomando como referencia el estándar ISO/IEC 9126. Los atributos de calidad comunes en los tres trabajos son eficiencia, productividad y eficacia, sin embargo, para Franke et al. (2012) la inclusión de los atributos flexibilidad, adaptabilidad, portabilidad, usabilidad y persistencia, son claves para realizar análisis comparativos entre aplicaciones móviles sobre un conjunto de criterios que brinden un mejor cubrimiento del potencial de estas aplicaciones. No obstante, al momento de realizar las comparaciones, los autores recurren a las pruebas funcionales de la aplicación y a la percepción del usuario al utilizar las aplicaciones, finalmente definen como parte del trabajo futuro el establecimiento de medidas específicas para medir los atributos de calidad del modelo propuesto. 
Para Alnanih et al. (2013) los factores adicionales a tener en cuenta son seguridad, navegabilidad y satisfacción, siendo éste último netamente subjetivo. Un punto diferenciador en este trabajo es la forma de validación del modelo, utilizando dos técnicas: validación teórica y validación empírica. En la primera técnica los autores tomaron como referencia la interpretación que hace el estándar ISO 9126-4 a los atributos de calidad, y asignaron medidas para realizar las mediciones, la validación les permitió demostrar que las medidas del modelo, se integran a los atributos de calidad observados. La segunda técnica implicó pruebas de usabilidad de la aplicación Twiter en dos escenarios, interfaz de usuario de escritorio (DIU) e interfaz de usuario de dispositivo móvil (MUI), con el objetivo de medir los efectos de la aplicación de acuerdo a los factores de calidad del modelo propuesto.

El modelo de calidad propuesto por La y Kim (2013) define cinco pasos: El primero consiste en realizar una medición de la calidad del servicio realizando un monitoreo a todos los servicios del ecosistema. El segundo paso consiste en identificar si el ecosistema tiene problemas, para esto se realiza la comparación de los atributos de calidad con umbrales previamente definidos. El paso tres propone un plan de acción para corregir los problemas encontrados. El paso cuatro ejecuta los planes de acción propuestos, finalmente, el paso cinco actualiza los repositorios con la nueva configuración y documenta las acciones realizadas. Como elemento diferenciador, este modelo propone realizar la medición de los atributos de calidad, efectividad y productividad, los cuales son medidos por dos métricas: la medición de tiempos de las tareas y la medición de finalización de tareas. Las métricas mencionadas se utilizan en los siguientes tres niveles: 1) instanciación del servicio, 2) tipo de servicio y 3) ecosistema en su totalidad. La información presentada en el artículo sobre el modelo de calidad de los autores La y Kim (2013), permite inferir dos cosas, primero, el modelo se concentra en aspectos no funcionales y segundo, que el modelo es límitado. La limitación del modelo genera los siguientes cuestionamientos: ¿Los problemas de calidad se pueden resolver solo con una configuración? ¿No hay problemas que requieran revisión de código y en ocasiones hasta rediseño y/o reimplementación de los componentes?

A diferencia de los trabajos anteriores, Hammam y Senbel (2013) proponen un sistema de gestión de confianza teniendo en cuenta tres aspectos: la disponibilidad, considerada debido a que las nubes que hacen parte de las redes ad-hoc se componen principalmente de dispositivos móviles. La evaluación y calidad de la respuesta de los vecinos, analizada debido a que los datos se trasfieren de agentes cliente a agentes nube y viceversa (vecinos), a través de un camino formado por otros agentes clientes, y finalmente, la exhaustividad al calcular el valor de confianza para un nodo. El sistema fue construido utilizando la arquitectura PlanetCloud, está arquitectura define tres componentes principales: agente cliente, agente en la nube y los servidores de recursos distribuidos. Estos elementos constituyen un sistema de reputación de los servicios en nube utilizados por los dispositivos móviles, este sistema de reputación opera con base en agentes clientes instalados en los dispositivos móviles, un agente en la nube (que recolecta y consolida la información) y los servidores de recursos distribuidos que se encargan de mantener actualizados los datos de los clientes que se almacenan en los repositorios de datos. Como método para el cálculo de la reputación de los nodos disponibles en el sistema, los autores utilizaron el algoritmo EigenTrust definido por Kamvar et al. (2003). Teniendo en cuenta que el propósito del sistema propuesto, es evitar la participación de agentes clientes maliciosos que puedan afectar el rendimiento de la nube formada, para la validación del sistema de confianza, se aplicaron pruebas de rendimiento entre el sistema PlanetCloud y un sistema propuesto por los autores, las pruebas permitieron comparar las características de funcionamiento de los agentes clientes, teniendo en cuenta lo siguiente clasificación: buenos agentes, agentes maliciosos y agentes desconocidos.

Para finalizar esta categoría, se encontró un estudio (Hoang et al., 2012) que aborda los problemas de control de admisión y asignación de los recursos adaptativos para el funcionamiento de las aplicaciones móviles en la nube (puntos de acceso). En este trabajo se plantea una solución que optimiza el uso de recursos compartidos por parte de usuarios finales, buscando el cumplimiento de los requisitos de calidad de servicio al momento de ejecutar aplicaciones. Los puntos de acceso a la nube se gestionan como procesos de decisión semi-markovianos y posteriormente se transforman en un modelo de programación lineal para así buscar una solución óptima.

\section{Calidad en el proceso de desarrollo}

Esta categoría está integrada por doce estudios que abordan la temática general de desarrollo global de software - GSD (Global Software Development) (Domingo et al.,2010). De los doce estudios, cuatro orientan su trabajo hacia los procesos de desarrollo software en GSD. Tres agrupaciones están conformadas por dos estudios: atributos de calidad, herramientas software y desafíos en equipos GSD. Finalmente hay dos trabajos no agrupados que se definen por sus temáticas particulares: métricas y formación de recurso humano en GSD. A continuación, se presentan los análisis de cada agrupación. 


\section{Procesos de desarrollo software}

Los trabajos que abordan la temática GSD desde la perspectiva de los procesos de desarrollo en este apartado son los siguientes: Guha y Al-Dabass (2010) proponen un proceso de desarrollo software como una extensión del modelo ágil denominado Extreme Programming - $X P$-. El modelo está dividido en seis actividades: recolección de requerimientos, análisis, diseño, desarrollo, prueba y despliegue. Adicionalmente, incluyen dos roles principales: desarrollador de software y el proveedor de la nube. La novedad del modelo radica en la relación de los roles establecidos con cada una de las actividades, algo que no poseen los métodos tradicionales. Como método para la estimación del desarrollo del software proponen COCOMO (COnstructive COst MOdel), sin embargo, este modelo no es aplicable a desarrollos para la nube, por ende, los autores indican la necesidad de realizar una extensión que permita medir el esfuerzo de desarrollo. Patidar et al. (2011) presentan un análisis del impacto de la computación en la nube en la ingeniería del software, éste análisis les permite sugerir dos modelos en el desarrollo de aplicaciones empresariales; la antigua, caracterizada por un desarrollo lento, ineficiente y con recursos humanos centralizados; la nueva, enfocada al desarrollo rápido, eficiente y personalizado para la nube. Con relación a las conclusiones del estudio, coinciden con el trabajo realizado por Guha y Al-Dabass (2010), el cual fue analizado anteriormente.

La diferencia entre Richardson et al. (2012) y los dos trabajos anteriores radica en que éste estudio toma como referencia el Modelo de Madurez de la Capacidad CMMi (Capability Maturity Model Integration) (CMMI, 2010). Los autores orientan la investigación hacia la creación de un área de proceso con metas y prácticas específicas. El objetivo de la nueva área de proceso es mitigar los problemas relacionados con la distancia temporal, cultural, geográfica y lingüística y a su vez que satisfagan las necesidades complejas y cambiantes de la gestión global de software. La identificación de factores claves para proyectos orientados al GSD se realizó mediante el análisis de tres casos de estudio. Entre los factores más importantes sobresalen: la comunicación, la gestión de habilidades, el idioma, las herramientas de trabajo y el miedo. Posterior a la identificación de los factores, los autores plantean dos preguntas de investigación: Primera, ¿Cuáles son las amenazas que enfrentan los equipos de desarrollo globales si no se implementa GSD?, los autores dan respuesta a esta pregunta haciendo una revisión bibliográfica, acompañada de la experiencia de trabajos previos entre 1999 y 2007 y del análisis de los casos de estudio verificados en este proyecto. Segunda, ¿Se pueden integrar los resultados de la investigación al CMMi? Para dar respuesta a esta pregunta de investigación los autores hacen una comparación exhaustiva entre los factores identificados y las áreas de proceso definidas en CMMi, el resultado de éste análisis indica que CMMi no cubre muchos de los factores encontrados, por lo tanto, presentan una propuesta de área de proceso que podría adherirse a CMMi.

Finalmente Salger et al. (2010) resaltan la importancia de la especificación de requerimientos y su influencia en la fase de diseño. Plantea cuatro retos: a) apropiación de toda la estructura de la especificación y el conocimiento total de la terminología por parte del equipo de trabajo, b) la viabilidad de todos los requerimientos y de una arquitectura software, c) conocimiento de todos los paquetes a realizar desde el punto de vista de los desarrolladores y d) el cumplimento de los objetivos del proyecto en los términos de calidad definidos. Para superar los retos expuestos se propone un marco de trabajo compuesto por tres tipos de evaluación. La primera se denomina verificación de la especificación y busca analizar si los conceptos de la especificación y su estructura son adecuados y conocidos para los participantes en el proyecto. La segunda denominada acceso a la calidad, verifica la calidad de la especificación y la arquitectura software a utilizar.

\section{Atributos de calidad en el proceso de desarrollo software}

Los siguientes dos estudios profundizan sobre los atributos de calidad en GSD. Chauhan y Babar (2012), abordan el GSD para identificar atributos de calidad y soluciones arquitectónicas para que la nube pueda proporcionar herramientas como servicios al GSD. Los atributos de calidad propuestos por los autores son el resultado de una revisión bibliográfica que incluye atributos funcionales y no funcionales, entre los que se resaltan: a) soporte para múltiples organizaciones: este concepto se conoce en computación en la nube como Multi-tenencia (Azeez et al., 2010) y hace referencia al aislamiento entre los servicios y los datos que se suministran a los clientes que comparten una infraestructura en nube; b) mantenimiento de diferentes versiones de las herramientas: este atributo hace referencia a la posibilidad de proveer múltiples versiones de una herramienta de acuerdo a las necesidades de un cliente o un requerimiento puntual, c) combinación de múltiples herramientas: las herramientas suministradas deben cubrir todas las fases de un proceso de desarrollo, por lo tanto, las salidas de una fase serán las entradas de otra, lo que implica una combinación inherente entre las herramientas utilizadas, d) compatibilidad con herramientas comerciales: en la actualidad existen muchas herramientas propietarias y de código abierto que dan soporte a los procesos de desarrollo software, por ende se debe proveer la integración de herramientas de diferentes proveedores, e) disponibilidad de herramientas para trabajar con datos privados: este atributo hace referencia a la integración de la infraestructura TaaS con nubes privadas de almacenamiento, e) soporte para múltiples métodos de persistencia: este atributo es relevante en las interfaces integradas de desarrollo, pues son ellas las que 
proveen acceso a la capa de datos, f) acceso desde múltiples tipos de dispositivos: las herramientas suministradas deben estar clasificadas de acuerdo a las fases del ciclo de desarrollo, de esta forma se proveen servicios desde la elicitación de requerimientos hasta las fases de pruebas en múltiples dispositivos, g) cumplimiento del acuerdo de nivel de servicio: este atributo hace referencia a los niveles de calidad de servicio que se deben ofrecer ante el uso de herramientas en múltiples fases de un proyecto de desarrollo.

Chatzipetrou et al. (2011) presentan un estudio empírico organizado en 24 aspectos de calidad software. Los aspectos fueron seleccionados por los participantes mediante voto acumulativo, cada participante vota por un atributo de calidad estableciendo un peso, de esta manera los atributos con mayor peso acumulado son seleccionados. Los aspectos de calidad los clasifican en dos niveles: alto y bajo. El alto nivel se compone de tres aspectos, cada uno de ellos está compuesto por características de bajo nivel. El alto nivel denominado Características, está compuesto por los aspectos de bajo nivel: idoneidad, precisión, interoperabilidad y estandarización. El segundo aspecto de alto nivel es propiedades del sistema el cual está conformado por 19 aspectos de bajo nivel entre ellos: seguridad, madurez, recuperación, instalación, entre otros. El último aspecto de alto nivel es denominado restricciones en la gestión del proyecto y contiene dos aspectos de bajo nivel: tiempo y costo.

\section{Herramientas software}

En este apartado se agrupan dos trabajos que presentan resultados asociados a la construcción de herramientas. En primer lugar, Hou y Zhou (2010) presentan la construcción un portal sobre una grilla académica bajo el concepto de nube privada. Los autores empaquetan las aplicaciones que van a ser utilizadas por los usuarios finales utilizando el modelo de contenedores y servicios web de Java, de esta forma hacen el despliegue de las aplicaciones a ofrecer. Las entradas de las aplicaciones se realizan a través de archivos planos que son cargados al portal por una interfaz de usuario final accesible desde cualquier navegador. Como motor para el control de flujo de trabajo los autores utilizan ODE, (Apache, 2010). Luego de realizar los procesos el portal, entrega los resultados y provee la asesoría de un experto para el análisis de los datos como valor agregado. Como trabajo futuro los autores están interesados en desplegar la solución sobre máquinas virtuales y la utilización de otros servicios diferentes al Web como el caso del FTP (File Transfer Protocol).

Por su parte, Miyamoto et al. (2012) plantean la estandarización de la documentación y el establecimiento de reglas para el GSD, soportado en una herramienta que permita verificar la calidad de los documentos involucrados en un proyecto, el trabajo aborda los siguientes problemas: a) manejo de múltiples formatos de documentos: el $80 \%$ de los documentos con formatos de herramientas tipo office no son estructurados (Reinsel et al., 2007), b) verificación de la relación entre documentos: el uso de identificadores dentro de los documento facilita esta labor, sin embargo, esto implicaría la construcción de componentes para herramientas de oficina particulares, por lo tanto, no es una solución viable puesto que limitaría el alcance del proyecto solo a documentos ofimáticos, c) flexibilidad para la definición de reglas: no todos los documentos utilizados en un proyecto siguen plantillas definidas, esto implica la definición de convenciones flexibles para que la herramienta pueda verificar muchos tipos de casos, d) independencia del lenguaje: los documentos internos son gestionados bajo las políticas de la empresa, sin embargo, los documentos externos en muchos casos dependen del contexto del cliente, en este caso la independencia del lenguaje es clave, e) rapidez y precisión: en cuanto al rendimiento restan importancia al tiempo requerido para analizar los documentos, fundamentados en la potencia de los recursos computacionales existentes, en cuanto a la precisión, los resultados al momento de verificar los documentos pueden arrojar falsos positivos, es decir, un aparente error que no es un error y falsos negativos al no encontrar un error donde si existe, para la definición de las reglas, el estudio toma como referencia el estándar IEEE 830-1998 (IEEE, 1998). El escenario de prueba del estudio tiene por cliente una empresa japonesa y el desarrollo es realizado por personal en China y Japón. Los resultados presentados arrojan una precisión del $98.3 \%$ en la detección de errores, un $0.6 \%$ de falsos negativos y como tipo de error más encontrado sobresale Inconsistencias.

\section{Desafíos en GSD}

Hashmi et al. (2011) presentan los desafíos encontrados en los proyectos de software globalmente dispersos. Este trabajo expone las ventajas y los desafíos del GSD, buscando la reducción de costos y tiempo de desarrollo (Lanubile et al., 2003 y Setamanit et al., 2007) a través del uso de la computación en la nube. Para GSD se proponen cuatro desafíos de colaboración: geográficos, culturales, lingüísticos y temporales. Cada uno de los desafíos tiene asociados un conjunto de problemáticas que los definen y posibles soluciones desde la computación en la nube.

Para el desafío geográfico las problemáticas propuestas son: distancia, tiempo, transferencia de conocimiento y herramientas. Estas problemáticas impactan negativamente los proyectos en la planeación y la calidad. Para 
los autores, estas problemáticas se pueden mitigar con la disponibilidad que provee SaaS al disminuir instalaciones en cada sitio de desarrollo, la adaptación en tiempo de ejecución y la disponibilidad total de recursos requeridos por cada desarrollador. Para el desafío cultural los problemas principales son: distribución de trabajo desigual, la falta de confianza entre las partes y el miedo. El impacto negativo en los proyectos se refleja en el incremento de los costos y mala gestión de los desarrolladores. Aunque los autores indican que estos temas pueden ser solventados por la computación en la nube mediante una mejor distribución de las tareas y una gestión del proyecto más sencilla, no indican el cómo se llevaría a cabo esta labor, las apreciaciones se sustentan en las definiciones y ventajas de la computación en la nube. El desafío lingüístico plantea dos problemas, la frecuencia en la comunicación y la transferencia de conocimiento. Los proyectos se ven afectados por la pérdida en la calidad del proyecto, la gestión inefectiva del mismo y la invisibilidad de los desarrolladores. No es claro en este punto como la computación en la nube puede aportar a la solución de este desafío. Finalmente, el reto temporal involucra dos problemas, la falta de motivación y el riesgo. La pérdida de la calidad sigue siendo un factor reiterativo en todos los desafíos, también se afectan los proyectos por una pobre gestión de la configuración y por la pérdida de artefactos. El aporte de la computación en la nube a este problema se sustenta en la centralización de la información y la gestión del inventario de los servicios, todo lo que se realice se registra y por tanto puede ser gestionado.

Raj et al. (2013) presentan un análisis y reflexión del impacto de la computación en la nube en la ingeniería del software. Expone conceptos básicos de la nube y centra su atención en el desarrollo global de aplicaciones como estrategia para construir software orientado a la nube, con equipos de trabajo distribuidos en cualquier parte del mundo y sin limitaciones de idioma o cultura. Los autores hablan de una evolución y revolución en la ingeniería del software, debido a que los conceptos de orientación a servicios de la computación en la nube requieren de estilos de trabajos innovadores y adecuados. La complejidad de una aplicación para la nube es mayor y se requieren herramientas que permitan gestionar estos desarrollos, por el momento la ingeniería del software provee los métodos ágiles para soportar éstos desarrollos. Por lo anterior, el estudio plantea una combinación necesaria entre agilidad y la nube para crear una nueva generación de la ingeniería del software, que apoye cuatro factores claves: a) tiempo, costo y productividad, b) desarrolladores distribuidos, c) innovación rápida y d) incremento de la complejidad de los proyectos.

Además de lo expuesto, el trabajo presenta un análisis de la convergencia entre los servicios y la nube. Esta convergencia es planteada como la evolución de todo desarrollo de software hacia una orientación de servicio. El trabajo resalta los riesgos en las fases de diseño y desarrollo de software orientado a servicios, cuando es realizado por entidades diferentes, desconocidas y en sitios diferentes distribuidos geográficamente. En este punto aparece el concepto de clasificación de servicios como un elemento diferenciador para determinar cuál servicio es de mejor calidad.

Otro tema abordado en este trabajo es la fusión entre la arquitectura orientada por modelos - MDA (Model Driven Architecture) y los paradigmas de la nube. Como estándar de facto es relacionado el lenguaje de modelado unificado UML (Unified Modeling Language) el cual sirve como base para el diseño y posterior ejecución de procesos en lenguajes como BPML (Business Process Modeling Languaje) y BPEL (Business Process Execution Language). La ingeniería del software orientada por modelos es presentada como una excelente alternativa a la hora de realizar desarrollos para la nube, con éstas técnicas se podría automatizar el desarrollo de aplicaciones orientadas a servicios.

Finalmente, el trabajo presenta los desafíos de la ingeniería del software para adaptarse a dos enfoques de desarrollo, por un lado, el tradicional y por el otro el nuevo paradigma de la nube. Entre los principales retos se encuentran: a) composición del software: el desarrollo tradicional implica la construcción de componentes que ensamblados constituyen la aplicación, mientras que en la nube se debe seleccionar los construidos por terceras partes para constituir la aplicación. b) la programación orientada a API frente a la orientada a consultas: herramientas como MapReduce y streaming entre otras, requieren de los desarrolladores un estilo de procesamiento orientado a consultas más funcional para obtener información. c) disponibilidad de código fuente: en el escenario actual se tiene el código fuente a la mano, en la nube muchos de los desarrollos ya están hechos y no hay código fuente, puesto que son realizados por terceros, el reto consiste en la compresión total del sistema y de sus partes, d) modelo de ejecución: las aplicaciones tradicionales generalmente se ejecutan en una máquina, por el contrario en la nube se ejecutan regularmente en varios equipos, esto implica un reto interesante para el estado de la trazabilidad del componente ejecutado y su depuración. e) gestión de aplicaciones: la gestión del ciclo de vida de una aplicación tradicional es sencilla de manejar comparada con la gestión de un ciclo de vida a nivel mundial, colaborativo y basado en la nube.

\section{Métricas}

Grbac y Huljenić (2011), analizan el GSD desde el punto de vista de las métricas para la detección de defectos en organizaciones que participan en un proyecto GSD y cómo las diferencias encontradas impactan la calidad 
del producto. La base de este trabajo es la cantidad de defectos encontrados en el desarrollo (Bird et al.,2009), de esta forma analizan la densidad de los defectos. Los autores aclaran que esta métrica no les permitió identificar si los defectos encontrados se debían a un mal diseño, a problemas en la codificación o a problemas inherentes al proceso de detección de los defectos. El caso de estudio presentado involucra nueve empresas filiales de Ericsson y busca desarrollar 216 unidades de código que requieren modificaciones. Este estudio presenta dos hipótesis: 1) la media de la densidad de defectos en etapas tempranas es igual para todas las empresas que participan en el desarrollo, esta hipótesis es falsa para este trabajo en particular. 2) la media de la densidad de defectos en etapas tardías es igual para todas las empresas que participan en el desarrollo, esta hipótesis por su parte es parcialmente correcta puesto que la diferencia en las densidades encontradas es mínima. La conclusión del trabajo indica que la eficacia en la detección de los defectos en etapas iniciales o finales, no influye en la calidad final del producto, sin embargo, es un factor clave al momento de realizar análisis en los procesos de desarrollo.

\section{Formación de recurso humano en GSD}

En Colomo-Palacios y Soto-Acosta (2011), se presenta una definición del término Mentoring como un proceso de mejora relacionado con aspectos del ambiente profesional e índole personal. Esta definición está conforme a la definición propuesta por el Modelo de Madurez de la Capacidad People-CMM, Hefley y Miller (1995), el cual expresa que la tutoría consiste en la transferencia de lecciones aprendidas por una persona con competencias formadas y amplia experiencia, a otros individuos o grupos de trabajo. El objetivo del estudio es identificar los procesos en la gestión de calidad de software que pueden llegar a ser influenciados por la tutoría en equipos GSD, esto les permite crear un escalafón de procesos SQM (Software Quality Managment), dependiendo del mejoramiento obtenido luego del proceso de tutoría, con estos resultados los autores obtienen una escala Likert que les permite identificar el impacto de la tutoría en los procesos. Inicialmente seleccionaron un grupo base compuesto por 5 pares expertos. Posteriormente tres investigadores realizaron reuniones virtuales con los expertos organizando la agenda de la siguiente forma: En la primera parte de la sesión el entrevistado presentaba sus experiencias acerca de SQM, GSD y el uso de la tutoría. En la segunda parte, el entrevistado y entrevistador discutían sobre la importancia de los retos de SQM y clasificaban los procesos tomando como base la lista de procesos SQM definidos por IEEE12207.0-96 (IEEE y EIA, 1996). EI resultado directo de la entrevista permitió a los autores clasificar los procesos SQM con base en un factor de impacto establecido por el uso de la tutoría. Los procesos clasificados con su respectiva escala fueron: proceso de validación ( $2=$ medio), proceso de revisión ( $2=$ medio), proceso de verificación ( $2=$ =medio), proceso aseguramiento de la calidad (1=bajo), proceso de auditoría (1=bajo). Los autores concluyen que el impacto de la tutoría para adoptar procesos de calidad en GSD es limitado, esto contrasta con el hecho que las tutorías son una herramienta recomendada para implementar prácticas relacionadas con la calidad, Schneidewind (2002). El análisis de los autores indica que la atomicidad de los procesos SQM y la complejidad de una labor de tutoría en cada uno de ellos puede ser la razón de los resultados, por tal motivo, sugieren la aplicación de la tutoría con una estrategia de calidad orientada a toda la compañía, de tal forma que las normas y modelos se adapten para asegurar la calidad en cada uno de los procesos.

\section{Calidad del Servicio}

Esta categoría está conformada por seis subgrupos: el primero de ellos lo componen ocho artículos que abordan los atributos de calidad como eje principal, el segundo lo componen diez artículos que proponen la construcción de modelos de calidad, el tercero está compuesto por cinco artículos que plantean arquitecturas como herramientas para la medición de la calidad del servicio, el cuarto lo conforman cuatro artículos que proponen asignación de recursos en la nube, el quinto es un artículo que abordan la temática de los fallos y su implicación en la calidad del servicio, a continuación se presentan las temáticas importantes que se han estudiado dentro de esta categoría:

\section{Atributos de calidad del servicio}

El rendimiento, la disponibilidad y la seguridad son los atributos de calidad más comunes en los artículos de este subgrupo. Bao et al. (2010) proponen un marco de medición que proporciona información sobre la calidad de los servicios en la nube a proveedores y usuarios en tiempo real. Cuando la calidad de servicio no cumple lo que se pactó en los acuerdos de nivel de servicio, se disparan alarmas vía correo electrónico para cada una de las partes. Hershey et al. (2012) presentan un enfoque orientado a los proveedores de servicios en la nube, que les permita satisfacer los acuerdos de niveles de servicio pactados con los clientes, para esto definen el rendimiento en función de tres métricas: latencia, variación de las latencias y la cantidad de trabajo realizado en un período de tiempo. La seguridad está definida en función de tres métricas: autenticación, autorización-certificación y acreditación. La recolección de datos para verificar los dos atributos mencionados, así como sus indicadores es la puesta en marcha de una aplicación de procesamiento de transacciones en línea. 
Hu y Zhang (2013) abordan los siguientes atributos: a) simpatía, vista por los autores como la calidad de los servicios de apoyo tecnológico y la atención al cliente que brindan los proveedores de servicios. Este atributo tiene tres métricas, 1) existencia de sistemas de entrenamiento, 2) servicio personalizado, y 3) servicio al cliente. b) Capacidad de respuesta, las métricas asociadas son: 1) respuesta de escalabilidad dinámica, 2) tiempo de recuperación ante desastres, y 3) disponibilidad de tecnología para soporte. c) confiabilidad, también está definida por tres métricas: 1) disponibilidad elástica del servicio, 2) precisión del servicio, y 3) control de presupuestos. d) Flexibilidad, capacidad que tiene los usuarios finales para cambiar parámetros por defecto, sus métricas son: 1) acceso para ajustes multi-cliente, 2) asignación de recursos adicionales, 3) migración de datos. e) Seguridad, definida como los métodos de protección sistemática que utilizan los proveedores para evitar la pérdida de información y el colapso del sistema, sus métricas son: 1) copias de seguridad de los datos, 2) estrategia de recuperación de fallos, 3) auditorías de seguridad periódicas, 4) herramientas antivirus, 5) privacidad de los datos y 6) control de acceso. La validación de los atributos de calidad propuestos por los autores se realizó en dos etapas, por un lado, el juicio de expertos mediante el diligenciamiento de cuestionarios y por el otro la recolección de datos para construir matrices que permitieran analizar los datos.

A diferencia de los estudios anteriores, en donde se plantean explícitamente los atributos para evaluar la calidad del servicio, C. Huang et al. (2012) hacen una revisión bibliográfica que presenta seis retos que enfrenta un proveedor de servicios de telecomunicaciones para ofrecer servicios en la nube. Los retos presentados por los autores son: a) Privacidad y seguridad, b) continuidad del servicio, c) migración de los servicios, d) almacenamiento escalable, e) escalamiento de rápido de servicios y f) cambio organizacional. Los retos presentados por los autores son atributos de calidad utilizados en la medición de la calidad del servicio.

El objetivo del estudio de Kiruthika et al. (2012) es crear un sistema de gestión de conocimiento para almacenar tipos de defectos conocidos al usar aplicaciones web, con la capacidad de agregar defectos a medida que estos se vayan presentando por el uso de aplicaciones de comercio electrónico. Los autores toman como referencia la norma ISO 9126 para correlacionar los atributos de calidad con los tipos de defectos tipificados en los servidores web, de este análisis surgen cinco atributos de calidad que mapean los tipos de errores más comunes al utilizar aplicaciones web: rendimiento, mantenibilidad, disponibilidad, seguridad y corrección. Krebs et al. (2013) definen el concepto de aislamiento de rendimiento como la capacidad que posee un sistema para no permitir que los clientes se vean afectados si las cuotas de rendimiento de otros clientes son excedidas. Con base en este concepto, los autores presentan dos grupos de métricas para cuantificar el aislamiento del rendimiento de los sistemas basados en la nube. El primer grupo denominado impacto de la calidad del servicio y el segundo grupo de métricas denominado puntos significativos.

Reynolds et al. (2011) tratan de definir una estrategia para predecir el rendimiento de un gran número de servicios. Los autores definen una norma de aprovisionamiento que calcula analíticamente la calidad de una configuración y la colocación de servicios en nodos de servidor. La norma ordena parcialmente todas las configuraciones posibles para un conjunto de nodos y servicios organizados, estableciendo una jerarquía en donde las primeras configuraciones son mejores que las últimas. La propuesta de la norma busca colocar los servicios eficiente y eficazmente en los nodos, de tal forma que el rendimiento sea altamente optimizado. Finalmente Hsueh et al. (2012) realizan una evaluación de la efectividad del servicio de computación en la nube basados en el marco de trabajo Hadoop, a partir de la capacidad de computación para grandes cantidades de datos. Realizan una recopilación de aplicaciones de código abierto que implementan patrones de diseño (Guerrero et al., 2013) y proporcionan a los programadores análisis de información en tiempo real que les ayuda a inspeccionar el valor de los patrones de diseño en el despliegue de las aplicaciones.

\section{Modelos de calidad}

Du y Li (2013) proponen un modelo de reputación para analizar la información histórica de los proveedores de servicios en la nube, de tal manera que le permita a un usuario seleccionar un proveedor de acuerdo a la confiabilidad de sus servicios. Como parte del modelo, los autores analizan tres atributos de calidad: seguridad, fiabilidad y disponibilidad. Los resultados del estudio permiten inferir un gran interrogante abierto: ¿Cuál es el incentivo para los proveedores de participar en este sistema? Por su parte, Liu et al. (2010) abordan el concepto de agentes para realizar mediciones en infraestructuras para la nube, en este trabajo los autores proponen que la medición de calidad debe realizarse en el momento de la ejecución del servicio y no en etapas previas o de pruebas. Un estudio similar es el de Garg et al. (2013); los autores proponen un marco de trabajo y un mecanismo que permite medir la calidad y priorización de los servicios de la nube, como una herramienta para que los consumidores puedan comparar los diferentes proveedores. Los autores estiman que el impacto será significativo, apoyando la sana competencia entre los proveedores de servicios en la nube. 
A diferencia de los tres trabajos anteriores, Massonet y Arenas (2012) no definen un modelo para evaluar calidad, los autores comparan la calidad de la protección de las aplicaciones analizando dos modelos: el modelo proveedor versus el modelo cliente. En primera instancia, el modelo proveedor publica un conjunto predefinido de opciones, posteriormente el cliente selecciona algunas de esas opciones, finalmente el cliente procede a realizar el despliegue de su aplicación en la nube del proveedor. En el modelo proveedor las opciones de calidad para la protección de aplicaciones son estandarizadas para todos los clientes.

El modelo de Qian et al. (2011) es teórico y se propone para evaluar la calidad de la experiencia de los servicios ofrecidos por un proveedor en la nube. De acuerdo al objetivo general de los autores el trabajo debería estar clasificado en la subcategoría denominada Calidad de la experiencia, sin embargo, el modelo propuesto por los autores analiza atributos de la calidad del servicio como: disponibilidad y latencia. El modelo de los autores define un valor máximo de referencia que permite a los proveedores informar a los usuarios la espera por un servicio.

J. Huang y Yanbing (2013) abordan el problema de cómo componer una secuencia de componentes de servicios para garantizar la calidad del servicio de aprovisionamiento en un ambiente de computación en la nube virtualizado. La contribución de la investigación incluye un modelo de aprovisionamiento de servicios para la nube y dos aproximaciones de algoritmos para QoS. Kumar et al. (2013) proponen un esquema de tres pasos para lograr la calidad de los servicios entregados por la nube: a) selección de recursos, b) programación de peticiones de usuarios con recursos compartidos y nueva asignación de recursos, y c) un algoritmo de programación de trabajos de adaptación. Para la planificación de tareas se define una métrica para programar de manera eficiente los trabajos que compiten por los recursos disponibles. La estrategia propuesta aumenta la fiabilidad de disponibilidad de recursos y reduce el tiempo de finalización del trabajo, aumentando la calidad en la entrega al usuario final.

Por su parte Kumar et al. (2013) realizan una discusión sobre varias alternativas para mapear requerimientos de una topología de clúster en ambientes de nube, para lograr alta confiabilidad y escalabilidad de aplicaciones ejecutadas dentro de recursos de la nube, habilitando la planificación para maximizar la CPU.

Más que un modelo, Li y Yu (2011) proponen el diseño e implementación de un servicio de seguimiento a la calidad de sistemas basados en computación en la nube, con el fin de mejorar la capacidad de vigilancia de las pequeñas y medianas empresas para el descubrimiento y solución oportuna de problemas con los servicios que se prestan a estas empresas. El sistema propuesto está desarrollado bajo tecnología SOA (Service Oriented Architecture).

Finalmente, en este apartado se contempla el estudio de Song et al. (2012), quienes proponen un modelo de evaluación basado en el sistema de evaluación de índices. Identifican los parámetros clave de calidad del servicio para determinar índices subjetivos y objetivos y el índice de terceras partes basado en el sistema de evaluación de índices.

\section{Arquitecturas como herramientas para la medición de la calidad del servicio}

El seguimiento de la calidad desde el punto de vista de las arquitecturas, es un área que ha dado origen a investigaciones que promueven estrategias y técnicas para gestionar la calidad. Marinescu y Petcu (2013) proponen una estrategia sobre cómo se podría emplear la infraestructura y los servicios que son desarrollos dentro del proyecto HOST para facilitar el uso extensivo de herramientas de evaluación de la calidad. El artículo presenta las mejoras en el rendimiento debido a la ejecución de perfusión dentro de una infraestructura de nube y se discuten las ventajas y desventajas de mover herramientas de análisis de software en la nube.

Boniface et al. (2010) proponen una nueva arquitectura PaaS para mejorar la Calidad del Servicio QoS en aplicaciones multimedia interactivas en línea. La arquitectura considera servicios como el de ingeniería de servicios, diseño de acuerdos de niveles de servicio, aprovisionamiento y monitoreo. La aplicabilidad genérica de la arquitectura es verificada y validada a partir de escenarios implementados en tres sectores de aplicación: post-producción de películas, realidad virtual aumentada, e-Learning de colaboración en mundos virtuales.

De otro lado, Hussain y Abdulsalam (2014) presentan una arquitectura para el diseño e implementación de servicios de certificación, para ofrecer servicios de seguimiento a las aplicaciones basadas en la nube para garantizar atributos de calidad del software como seguridad, privacidad, confianza y capacidad. El enfoque de la arquitectura se basa en el usuario para ofrecerles mayor control sobre sus propios datos y aplicaciones, en contraste con Sagbo y Houngue (2012) que proponen una arquitectura de asignación de recursos para computación en la nube que permita la medición de indicadores de calidad KPI (Key Performance Indicators). La arquitectura propone diferentes asignaciones de recursos: predictivo y reactivo. Las decisiones de asignación son tomadas en esta arquitectura tomando como referencia. 
Finalmente Shangguang Wang et al. (2012) proponen un enfoque de evaluación precisa de Calidad en Servicios de la Nube QCoS (Quality Cloud of Services), orientado a los servicios de computación en la nube. Inicialmente emplean decisión sintética difusa para evaluar a los proveedores de servicios en la nube de acuerdo a las preferencias de los usuarios en la nube y posteriormente adoptaron el modelo de nube para computación de incertidumbre de servicios en la nube.

\section{Algoritmos para la asignación de recursos en la nube}

Belalem et al. (2011) proponen una extensión del simulador CloudSim a partir de los algoritmos heredados del simulador GridSim. Los algoritmos de referencia no soportan la virtualización, por lo tanto, los autores introducen parámetros y funciones para adaptarlo al ambiente de computación en la nube. En SX Wang et al. (2010) los autores analizan las características importantes de la confianza y los factores clave que afectan a la relación de confianza en el entorno de los servicios Web, con el fin de proponer un modelo de confianza basado en la teoría del modelo de nube para describir las incertidumbres subjetivas de los factores de confianza. Adicionalmente, aplican un algoritmo para expresar el dinamismo de la confianza. Como resultado proporcionan un método de cálculo formalizado para evaluar el grado de confianza de los solicitantes de servicios de los proveedores.

Zheng et al. (2013) proponen un marco de trabajo para la predicción de la Calidad del Servicio QoS, para servicios en la nube basado en el aprovechamiento de la experiencia de usos pasados de los servicios por otros clientes. Finalmente, el algoritmo de You et al. (2011) soporta una estrategia automática para la asignación de los recursos basada en los mecanismos del mercado.

\section{Fallos y su implicación en la calidad del servicio}

Sun et al. (2013) presentan en primera instancia las definiciones de fallos, errores, y los fracasos que ocurren en los servicios de la nube y posteriormente proponen los principios que se deben tener en cuenta para definir los objetivos de alta tolerancia a fallos, utilizan como línea de base la estrategia DAFT (Dynamic Adaptive Fault Tolerance)

\section{AMENAZAS DE LA VALIDEZ DEL ESTUDIO}

Es importante mencionar algunos aspectos que pueden afectar la validez del presente estudio: Durante el proceso de búsqueda por palabras claves es posible que se hayan omitido algunas fuentes, debido a que las palabras claves utilizadas por algunos autores son sinónimos de las utilizadas en el presente estudio. Aunque se tuvieron en cuenta variaciones de las palabras claves, es posible que no se cubrieran todas.

Otro aspecto relevante es la ventana de observación de los trabajos analizados, la fecha de cierre de la ventana de observación excluyó gran cantidad de artículos del año 2014 que aún no habían sido publicados. La tendencia para ese año indica que la cantidad de artículos debía ser superior a 77 . Fue necesario realizar el corte a esa fecha con el fin de consolidar una base de información estable, que permitiera iniciar el análisis de las fuentes. En la actualidad, se está realizando otro estudio que incluye la totalidad del año 2014 y el año 2015 con corte a julio.

\section{CONCLUSIONES}

El presente estudio es una ampliación del trabajo realizado por Singh y Chana (2012), permitió identificar nuevas categorías para agrupar los trabajos desarrollados en el contexto de computación en la nube. Por lo tanto, este nuevo estudio puede convertirse en una fuente primaria para trabajos posteriores en esta temática. Este análisis le presenta al lector múltiples problemas abiertos que pueden ser la base para futuros trabajos de investigación de nivel de maestría y doctorado.

A pesar de la existencia de atributos de calidad y de modelos para medir la calidad del servicio, difícilmente se pueden aplicar a proveedores privados, esto implica que para el cliente exista un modelo de caja negra al momento de establecer los acuerdos de niveles de servicio. Los modelos analizados en este estudio están orientados a proveedores públicos en procesos de prestación de servicio ideal. La realidad es que la computación en la nube está dominada por 6 proveedores mundiales, los cuales están en mora de proponer un único modelo para garantizar a sus usuarios la calidad del servicio que se exige.

Las metodologías de desarrollo tradicional están siendo adaptadas a procesos de desarrollo para la computación en la nube, sin embargo, aún no es clara la participación de los proveedores en los procesos de desarrollo orientados a la nube. El acompañamiento del proveedor debería iniciar en la fase de levantamiento de requerimientos y no al final cuando la aplicación ya ha sido desarrollada. 
La computación móvil es uno de los principales clientes de los servicios provistos en la nube, por lo tanto, la medición de requerimientos no funcionales impacta de manera directa los requerimientos funcionales, enfocando los desarrollos dentro de los contextos de ecosistemas digitales.

La calidad de los datos y la privacidad de los mismos siguen y seguirán siendo un tema crítico en los niveles de acuerdo de servicio, aspectos como el rendimiento y disponibilidad de los datos, aún deben correlacionarse con variables como la transferencia y la rapidez en la recuperación de los mismos.

\section{REFERENCIAS}

Abdullah, R., Eri, Z. D. y Talib, A. M., A model of knowledge management system for facilitating knowledge as a service (KaaS) in cloud computing environment, doi: 10.1109/ICRIIS.2011.6125691, International Conference on Research and Innovation in Information Systems. 1-4 (2011)

Alnanih, R., Ormandjieva, O. y Radhakrishnan, T., A New Quality-in-Use Model for Mobile User Interfaces, doi: 10.1109/IWSM-Mensura.2013.32, Conference of the 23rd Intern. Workshop on Software Measurement and the 8th International Conference on Software Process and Product Measurement. 165-170 (2013)

Apache. Apache ODE [En línea] (2010) http://ode.apache.org/ Acceso: 20 de marzo de 2014.

Ardagna, C. A., Damiani, E., Frati, F., Rebeccani, D. y Ughetti, M., Scalability Patterns for Platform-as-aService, doi: 10.1109/CLOUD.2012.41, Fifth International Conference on Cloud Computing. 718-725 (2012)

Azeez, A., Perera, S., Gamage, D., Linton, R., Siriwardana, P., Leelaratne, D. y Fremantle, P. Multi-tenant SOA Middleware for Cloud Computing, doi: 10.1109/CLOUD.2010.50, International Conference on Cloud Computing. 458-465 (2010)

Bao, D., Xiao, Z., Sun, Y. y Zhao, J. A method and framework for quality of cloud services measurement, doi: 10.1109/ICACTE.2010.5579535. International Conference on Advanced Computer Theory and Engineering(ICACTE). 358-362 (2010)

Belalem, G., Bouamama, S. y Sekhri, L., An Effective Economic Management of Resources in Cloud Computing, 10.4304/jcp.6.3.404-411, Journal of Computers. 6(3), 404-411 (2011)

Bird, C., Nagappan, N., Devanbu, P., Gall, H. y Murphy, B., Does distributed development affect software quality? An empirical case study of Windows Vista, doi: 10.1109/ICSE.2009.5070550, IEEE 31st International Conference on Software Engineering. 518-528 (2009)

Boniface, M., Nasser, B., Papay, J., Phillips, S. C., Servin, A. y ang, X. y Kyriazis, D. (2010). Platform-as-aService Architecture for Real-Time Quality of Service Management in Clouds, doi: 10.1109/ICIW.2010.91, Fifth International Conference on Internet and Web Applications and Services. 155-160 (2010)

Chatzipetrou, P., Angelis, L., Barney, S. y Wohlin, C., Software Product Quality in Global Software Development: Finding Groups with Aligned Goals, doi: 10.1109/SEAA.2011.67, 37th EUROMICRO Conference on Software Engineering and Advanced Applications. 435-442 (2011)

Chauhan, M. A. y Babar, M. A., Cloud infrastructure for providing tools as a service, doi: 10.1145/2361999.2362002, Proceedings of the WICSA/ECSA 2012 Companion. 5-13 (2012)

CMMI. CMMI for Development, Version 1.3. [En línea], 2010. Carnegie Mellon University, https://books.google.com.co/books?hl=en\&lr=\&id=vEOsAgAAQBAJ\&oi=fnd\&pg=PA1\&dq=CMMl\%C2\%AE+f or+Development,+Version+1.3\&ots=apjv1KKyAl\&sig=yL4ZsMk0A0UMuZQZ9VeO4SFKD2w\&redir_esc=y\#v $=$ onepage \&q=CMMI\%C2\%AE\%20for\%20Development\%2C\%20Version\%201.3\&f=false. Acceso: 25 de febrero de 2014

Colomo-Palacios, R. y Soto-Acosta, P., Software quality management improvement through mentoring: an exploratory study from GSD projects, doi: 10.1007/978-3-642-25126-9_29, The Move to Meaningful Internet Systems: OTM 2011 Workshops. 190-199 (2011)

Comerio, M., Truong, H.-L., Batini, C. y Dustdar, S., Service-oriented data quality engineering and data publishing in the cloud, doi: 10.1109/SOCA.2010.5707184, International Conference on Service-Oriented Computing and Applications (SOCA). 1-6 (2010)

Domingo, E. J., Nino, J. T., Lemos, A. L., Lemos, M. L., Palacios, R. C. y Berbís, J. M. G., CLOUDIO: A Cloud Computing-Oriented Multi-tenant Architecture for Business Information Systems, doi: 10.1109/CLOUD.2010.88, 3rd International Conference on Cloud Computing. 532-533 (2010) 
$\mathrm{Du}, \mathrm{J}$ y Li, X., Adaptive and attribute-based trust model for service-level agreement guarantee in cloud computing, doi: 10.1049/iet-ifs.2012.0232, IET Information Security. 7(1), 39-50 (2013)

Franke, D., Kowalewski, S. y Weise, C., A Mobile Software Quality Model, doi: 10.1109/QSIC.2012.49, 12th International Conference on Quality Software. 154-157 (2012)

Garg, S. K., Versteeg, S. y Buyya, R. A framework for ranking of cloud computing services, doi: 10.1016/j.future.2012.06.006, Future Generation Computer Systems. 29(4), 1012-1023 (2013)

Grbac, T. y Huljenić, D., Defect Detection Effectiveness and Product Quality in Global Software Development, doi: 10.1007/978-3-642-21843-9, Product-Focused Software Process Improvement. 113-127 (2011)

Guerrero, C. A., Suárez, J. M. y Gutiérrez, L. E., Patrones de Diseño GOF (The Gang of Four) en el contexto de Procesos de Desarrollo de Aplicaciones Orientadas a la Web, doi: 10.4067/S0718-07642013000300012, Información Tecnológica. 24(3), 103-114 (2013)

Guha, R. y Al-Dabass, D., Impact of Web 2.0 and Cloud Computing Platform on Software Engineering, doi: 10.1109/ISED.2010.48, International Symposium on Electronic System Design. 213-218 (2010)

Guo, J., An approach for modeling and designing software architecture, doi: 10.1109/ECBS.2003.1194787, 10th IEEE Intern. Conference and Workshop on the Eng. of Computer-Based Systems. 89-97 (2003)

Hammam, A. y Senbel, S., A trust management system for ad-hoc mobile clouds, doi: 10.1109/ICCES.2013.6707167, 8th Inter. Conference on Computer Eng. \& Systems (ICCES). 31-38 (2013)

Hashmi, S. I., Clerc, V., Razavian, M., Manteli, C., Tamburri, D. A., Lago, P. y Richardson, I. Using the Cloud to Facilitate Global Software Development Challenges, doi: 10.1109/ICGSE-W.2011.19, IEEE Sixth International Conference on Global Software Engineering Workshop. 70-77 (2011)

Hefley, B. y Miller, S., People Capability Maturity Model (P-CMM) [En línea], (1995). http://oai.dtic.mil/oai/oai?verb=getRecord\&metadataPrefix=html\&identifier=ADA512354. Acceso: 20 de noviembre de 2013.

Hershey, P., Rao, S., Silio, C. B. y Narayan, A., System of Systems to provide Quality of Service monitoring, management and response in cloud computing environments, doi: 10.1109/SYSoSE.2012.6384208, 7th International Conference on System of Systems Engineering (SoSE). 314-320 (2012)

Hoang, D. T., Niyato, D. y Wang, P., Optimal admission control policy for mobile cloud computing hotspot with cloudlet, doi: 10.1109/WCNC.2012.6214347, IEEE Wireless Communications and Networking Conference (WCNC). 3145-3149 (2012)

Hobfeld, T., Schatz, R., Varela, M. y Timmerer, C., Challenges of QoE management for cloud applications, doi: 10.1109/MCOM.2012.6178831, IEEE Communications Magazine. 50(4), 28-36 (2012)

Hou, Z. y Zhou, X., ASAAS: Application Software as a Service for High Performance Cloud Computing, doi: 10.1109/HPCC.2010.76, 12th International Conference on High Performance Computing and Communications (HPCC). 156-163 (2010)

Hsueh, N.-L., Wang, J.-Y. y Ting, D.-H., A Cloud Service Design for Quality Evaluation of Design Patterns, doi: 10.1109/ICGEC.2012.9, Sixth International Conference on Genetic and Evolutionary Computing. 184-187 (2012)

Hu, H. y Zhang, J., The Evaluation System for Cloud Service Quality Based on SERVQUAL, doi: 10.1007/9783-642-34528-9, Proceedings of the 2012 International Conference on Information Technology and Software Engineering. 577-584 (2013)

Huang, C., Hsu, P. y Tzeng, G., Evaluating cloud computing based telecommunications service quality enhancement by using a new hybrid MCDM model, doi: 10.1007/978-3-642-29977-3_52, Intelligent Decision Technologies. 1, 519-536 (2012)

Huang, J. y Yanbing, L. Modeling and algorithms for QoS-aware service composition in virtualization-based cloud computing [En línea], (2013). IEICE Transactions and Communications. http://search.ieice.org/bin/summary.php?id=e96-b_1_10. Acceso: 25 de noviembre de 2013.

Hussain, M. y Abdulsalam, H. M., Software quality in the clouds: a cloud-based solution, doi: 10.1007/s10586012-0233-8, Cluster Computing. 17(2), 389-402 (2012) 
IEEE., IEEE Recommended Practice for Software Requirements Specifications, [En línea], 1998. http://www.math.uaa.alaska.edu/ afkjm/cs401/IEEE830.pdf. Acceso: 25 de febrero de 2014.

IEEE, y EIA., Industry Implementation of International Standard ISO/IEC 12207: 1995, (ISO/IEC 12207), doi: SH94581, Standard for Information Technology-Software life cycle processes. (1996)

Kamvar, S. D., Schlosser, M. T. y Garcia-Molina, H., The Eigentrust algorithm for reputation management in P2P networks, doi: 10.1145/775152.775242, Proceedings of the twelfth international conference on World Wide Web - WWW '03. 640 (2003)

Kiruthika, J., Horgan, G. y Khaddaj, S., Quality Measurement for Cloud Based E-commerce Applications, doi: 10.1109/DCABES.2012.62, 11th International Symposium on Distributed Computing and Applications to Business, Engineering \& Science. 209-213 (2012)

Klems, M., Bermbach, D. y Weinert, R., A Runtime Quality Measurement Framework for Cloud Database Service Systems, doi: 10.1109/QUATIC.2012.17, Eighth International Conference on the Quality of Information and Communications Technology. 38-46 (2012)

Krebs, R., Momm, C. y Kounev, S., Metrics and techniques for quantifying performance isolation in cloud environments, doi: 10.1016/j.scico.2013.08.003, Science of Computer Programming. 1, 1-19 (2013)

Kumar, N., Chilamkurti, N., Zeadally, S. y Jeong, Y.-S., Achieving Quality of Service (QoS) Using Resource Allocation and Adaptive Scheduling in Cloud Computing with Grid Support, doi: 10.1093/comjnl/bxt024, The Computer Journal. 57(2), 281-290 (2013)

La, H. J. y Kim, S. D., A model of quality-in-use for service-based mobile ecosystem, doi: 10.1109/MOBS.2013.6614217, 1st International Workshop on the Engineering of Mobile-Enabled Systems (MOBS). 13-18 (2013)

Lanubile, F., Damian, D. y Oppenheimer, H., Global software development: technical, organizational, and social challenges. [En línea], 2003. ACM SIGSOFT Software, 28(6), 1-4. http://dl.acm.org/citation.cfm?id=966224. Acceso: 10 de diciembre de 2013.

Li, X. y Yu, Q., Design and implementation of quality tracking system based on cloud computing, doi: 10.1109/ICCSNT.2011.6182476, Proceedings of 2011 International Conference on Computer Science and Network Technology. 2495-2498 (2011)

Liao, W.-H. y Su, S.-C., A Dynamic VPN Architecture for Private Cloud Computing, doi: 10.1109/UCC.2011.68, Fourth IEEE International Conference on Utility and Cloud Computing. 409-414 (2011)

Liu, Z., Liu, T., Lu, T., Cai, L. y Yang, G., Agent-Based Online Quality Measurement Approach in Cloud Computing Environment, doi: 10.1109/WI-IAT.2010.213, IEEE/WIC/ACM International Conference on Web Intelligence and Intelligent Agent Technology. 686-690 (2010)

Marinescu, C. y Petcu, D. Quality Assessment in the Cloud: Is It Worthwhile?, doi: 10.1109/CSMR.2013.70, 17th European Conference on Software Maintenance and Reengineering. 453-456 (2013)

Massonet, P. y Arenas, A., Towards more Transparency in Quality of Protection for Infrastructure Clouds, doi: 10.1109/QUATIC.2012.62, Eighth International Conference on the Quality of Information and Communications Technology, 285-290 (2012)

Miyamoto, K., Nerome, T. y Nakamura, T., Document Quality Checking Tool for Global Software Development, doi: 10.1109/SRII.2012.37, Annual SRII Global Conference, 267, 276 (2012)

Patidar, S., Rane, D. y Jain, P., Challenges of software development on cloud platform, doi: 10.1109/WICT.2011.6141386, World Congress on Information and Comm. Technologies. 1009-1013 (2011)

Qian, H., Medhi, D. y Trivedi, K., A hierarchical model to evaluate quality of experience of online services hosted by cloud computing, doi: 10.1109/INM.2011.5990680, 12th IFIP/IEEE International Symposium on Integrated Network Management (IM 2011) and Workshops. 105-112 (2011)

Raj, P., Venkatesh, V. y Amirtharajan, R., Envisioning the Cloud-Induced Transformations in the Software Engineering Discipline, doi: 10.1007/978-1-4471-5031-2. Software Engineering Frameworks for the Cloud Computing Paradigm. 25-53 (2013)

Reinsel, D., Chute, C. y Schlichting, W., The Expanding Digital Universe. White Paper, IDC. 2007 
Reynolds, M. B., Hopkinson, K. M., Oxley, M. E. y Mullins, B. E., Provisioning Norm: An Asymmetric Quality Measure for SaaS Resource Allocation, doi: 10.1109/SCC.2011.16, IEEE International Conference on Services Computing. 112-119 (2011)

Richardson, I., Casey, V., McCaffery, F., Burton, J. y Beecham, S., A Process Framework for Global Software Engineering Teams, doi: 10.1016/j.infsof.2012.05.002, Information and Software Technology. 54(11), 11751191 (2012)

Sagbo, K. y Houngue, P., Quality architecture for resource allocation in cloud computing, doi: 10.1007/978-3642-33427-6_11, Service-Oriented and Cloud Computing. 154-168 . (2012)

Salger, F., Engels, G. y Hofmann, A., Assessments in global software development, doi: 10.1145/1810295.1810301, Proceedings of the 32nd ACM/IEEE International Conference on Software Engineering - ICSE '10. Vol. 2, 29-38 (2010)

Schneidewind, N. E., Body of knowledge for software quality measurement, doi: 10.1109/2.982919, Computer, 35(2), 77-83 (2002)

SJR. SCImago Journal \& Country Rank. [En línea], 2014. ScimagoLab. www.scimagojr.com/. Acceso: 15 de noviembre de 2013.

Setamanit, S., Wakeland, W. y Raffo, D., Improving Global Software Development Project Performance Using Simulation, doi: 10.1109/PICMET.2007.4349580, PICMET '07 - 2007 Portland International Conference on Management of Engineering \& Technology. 2458-2466 (2007)

Singh, S. y Chana, I., Cloud Based Development Issues: A Methodical Analysis, doi: 10.11591/closer.v2i1.1704, International Journal of Cloud Computing and Services Science (IJ-CLOSER). 2(1), 73-84 (2012)

Song, J., Zhang, S., Gong, Y. y Dai, B., A QoS Evaluation Model for Test-Bed in the Cloud Computing Environment, doi: 10.1109/ICEBE.2012.54, IEEE Ninth International Conference on e-Business Engineering. 292-295 (2012)

Sun, D., Chang, G., Miao, C. y Wang, X., Analyzing, modeling and evaluating dynamic adaptive fault tolerance strategies in cloud computing environments, doi: 10.1007/s11227-013-0898-7, The Journal of Supercomputing. 66(1), 193-228 . (2013)

Wang, S., Liu, Z., Sun, Q., Zou, H. y Yang, F., Towards an accurate evaluation of quality of cloud service in service-oriented cloud computing, doi: 10.1007/s10845-012-0661-6, Journal of Intelligent Manufacturing. 25(2), 283-291 (2012)

Wang, S., Zhang, L., Wang, S. y Qiu, X., A cloud-based trust model for evaluating quality of web services, doi: 10.1007/s11390-010-1090-7, Journal of Computer Science and Technology. 25(6), 1130-1142 (2010)

Yang, K. y Jia, X., Data storage auditing service in cloud computing: challenges, methods and opportunities, doi: 10.1007/s11280-011-0138-0, World Wide Web. 15(4), 409-428 (2011)

You, X., Wan, J., Xu, X., Jiang, C., Zhang, W. y Zhang, J., ARAS-M: Automatic Resource Allocation Strategy based on Market Mechanism in Cloud Computing, doi: 10.4304/jcp.6.7.1287-1296, Journal of Computers. 6(7), 1287-1296 (2011)

Yu, L., Tsai, W.-T., Chen, X., Liu, L., Zhao, Y., Tang, L. y Zhao, W., Testing as a Service over Cloud, doi: 10.1109/SOSE.2010.36, Fifth IEEE International Symposium on Service Oriented System Engineering. 181$188(2010)$

Zheng, Z., Wu, X., Zhang, Y., Lyu, M. R. y Wang, J., QoS Ranking Prediction for Cloud Services, doi: 10.1109/TPDS.2012.285, IEEE Transactions on Parallel and Distributed Systems. 24(6), 1213-1222 (2013)

Zrnec, A. y Lavbič, D., Comparison of Cloud vs. Tape Backup Performance and Costs with Oracle Database, [En línea], (2011). Journal of Information and Organizational Sciences. 35(1), 135-142. http://hrcak.srce.hr/file/103900. Acceso: 27 de enero de 2014. 
